Article

\title{
Arenobufagin Induces Apoptotic Cell Death in Human Non-Small-Cell Lung Cancer Cells via the Noxa-Related Pathway
}

\author{
Liang Ma, Yindi Zhu, Sheng Fang, Hongyan Long, Xiang Liu * and Zi Liu * \\ Department of Chemical Biology and Pharmaceutical Engineering, School of Chemistry and Chemical \\ Engineering, Anhui University of Technology, Ma'anshan 243002, Anhui, China; alexingma@163.com (L.M.); \\ zhuyindi0805@163.com (Y.Z.); fngseng12345@163.com (S.F.); longhongyan1995@163.com (H.L.) \\ * Correspondence: liuxiang@ahut.edu.cn (X.L.); tarring126@126.com (Z.L.); Tel.: +86-555-231-1551 (Z.L.)
}

Received: 9 August 2017; Accepted: 8 September 2017; Published: 11 September 2017

\begin{abstract}
Arenobufagin, an active component isolated from the traditional Chinese medicine Chan $\mathrm{Su}$, exhibits anticancer influences in several human malignancies. However, the effects and action mechanisms of arenobufagin on non-small-cell lung cancer (NSCLC) are still unknown. In this study, we reported that arenobufagin acted through activation of Noxa-related pathways and promoted apoptotic cell death in human NSCLC cells. Our results revealed that arenobufagin-induced apoptosis was caspase-dependent, as evidenced by the fact that caspase-9, caspase- 3 and poly (ADP-ribose) polymerase (PARP) were cleaved, and pretreatment with a pan-caspase inhibitor Z-VAD-FMK inhibited the pro-apoptosis effect of arenobufagin. Mechanistically, we further found that arenobufagin rapidly upregulated the expression of the pro-apoptosis protein Noxa, and abrogated the anti-apoptosis protein Mcl-1, a major binding partner of Noxa in the cell. More importantly, the knockdown of Noxa greatly blocked arenobufagin-induced cell death, highlighting the contribution of this protein in the anti-NSCLC effects of arenobufagin. Interestingly, arenobufagin also increased the expression of p53, a direct transcriptional activator for the upregulation of the Noxa protein. Taken together, our results suggest that arenobufagin is a potential anti-NSCLC agent that triggers apoptotic cell death in NSCLC cells through interfering with the Noxa-related pathway.
\end{abstract}

Keywords: non-small-cell lung cancer; arenobufagin; Noxa; Mcl-1; p53

\section{Introduction}

Lung cancer continues to be the most common cause of cancer death in the world, responsible for nearly one cancer death in five [1]. Non-small-cell lung cancer (NSCLC), which primarily includes adenocarcinoma and squamous cell carcinoma, accounts for approximately $85 \%$ of all cases of lung cancer. Despite advances in early detection, NSCLC is often diagnosed at an advanced stage, or a locally advanced stage. Chemotherapeutic agents have provided the standard regimen backbone for these patients, as well as combination chemotherapy. However, as a result of resistance, the prognosis for NSCLC patients is still poor, with a 5-year survival rate of less than $15 \%[2,3]$. Therefore, there is an urgent need to develop more effective therapies for NSCLC patients.

Apoptosis pathways are typically dysregulated in many human cancers and can be therapeutically exploited for cancer treatment. In the past decade, the discovery and development of novel small-molecule inhibitors targeting apoptosis have been widely reported. There are two major apoptotic mechanisms: the intrinsic (mitochondrial) and the extrinsic (death receptor), which are tightly regulated by the balance between pro- and anti-apoptotic proteins [4]. The Bcl-2 protein family, including anti-apoptotic proteins (i.e., Bcl-2, Bcl-xl, BCL-w, Bcl-b, Mcl-1, and A1/Bfl-1) and two groups of pro-apoptotic proteins: multi-domain proteins (e.g., Bak, Bax) and BH3-only proteins (i.e., Bim, 
Bid, Bad, Puma, Bmf, Bik, Hrk, and Noxa), are crucial in regulating mitochondrial apoptosis [5]. Noxa was initially identified as a primary p53-responsive gene [6]. Recently, accumulating evidence indicates that Noxa is involved in the regulation of the cytotoxic effect triggered by a plethora of anticancer treatments [7-9]. Independent of its inherent pro-apoptotic activity, it was reported that Noxa played a critical role in regulating Mcl-1, an anti-apoptotic member of the Bcl-2 proteins family that is overexpressed in many tumor types [10-13]. Thus, activating Noxa-related pathways may represent a new treatment strategy for cancer.

Anti-cancer agents from natural products used in traditional Chinese medicines have attracted significant attention internationally [14]. Arenobufagin, one of the active ingredients of toad venom (also called Chan $\mathrm{Su}$ ), is a traditional Chinese medicine obtained from the skin and parotid venom glands of the toad $[15,16]$. Arenobufagin was initially identified as a potent $\mathrm{Na}^{+} / \mathrm{K}^{+}$pump inhibitor, and has the ability to block $\mathrm{Na}^{+}-\mathrm{K}^{+}$pumps in cardiac myocytes $[17,18]$. In recent years, researchers found that arenobufagin possessed the potential for anti-tumor activity in some malignances. For instance, Zhang et al. reported that arenobufagin induced apoptosis and autophagy in human hepatocellular carcinoma (HCC) cells, via inhibition of the PI3K/Akt/mTOR pathway [19]. Deng et al. found that arenobufagin could intercalate with DNA to induce G2 cell cycle arrest through the ATM/ATR pathway in HCC cells [20]. Except in HCC, arenobufagin has also been shown to inhibit growth and induce apoptosis in human esophageal squamous cell carcinoma cells and cervical carcinoma cells $[21,22]$. However, the effects and mechanisms of arenobufagin on lung cancer are still not clear.

Here, we systematically evaluated the anti-cancer effect of arenobufagin on NSCLC cells. Our data demonstrated that arenobufagin significantly inhibited growth and induced apoptosis of NSCLC cells. More importantly, we reported a novel finding that activating Noxa-related pathways was critical in arenobufagin-triggered cell death. These results suggested that arenobufagin could be a promising agent for patients with NSCLC.

\section{Results}

\subsection{Effects of Arenobufagin on NSCLC Cells}

We tested the effects of arenobufagin (Figure 1A) on multiple NSCLC cells and normal human bronchial epithelial cells. The results showed that arenobufagin exhibited higher activity to A549, NCI-H460 and NCI-H1975 NSCLC cells, with IC $_{50}$ values around $10 \mathrm{nM}$. Arenobufagin displayed less sensitivity towards $16 \mathrm{HBE}$ normal human bronchial epithelial cells (more than $40 \mathrm{nM}$ of the $\mathrm{IC}_{50}$ value) (Figure 1B), indicating its selectivity between cancer and normal cells. Thereafter, we systematically evaluated the effect of arenobufagin on $\mathrm{A} 549$ and NCI-H460 cells with lower $\mathrm{IC}_{50}$ values (Figure 1B). Our results showed that arenobufagin significantly inhibited the growth of A549 and NCI-H460 cells in a time- and dose-dependent manner by 3-(4,5-dimethylthiazol-2-yl)-2, 5-diphenyltetrazolium bromide (MTT) assay (Figure 1C,D). Trypan blue exclusion assay further demonstrated that arenobufagin reduced viable cells in A549 and NCI-H460 (Figure 1E,F). Similar results were also observed in NCI-H1975 cells (Supplementary, Figure S1A,B). These results indicated that arenobufagin exhibits great therapeutic potential in NSCLC treatment. 
A

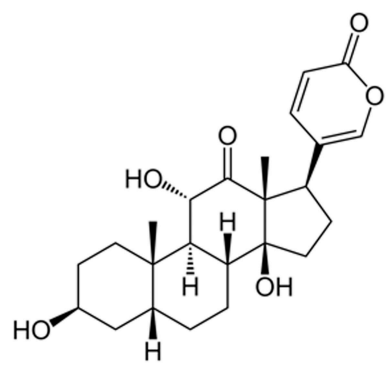

C

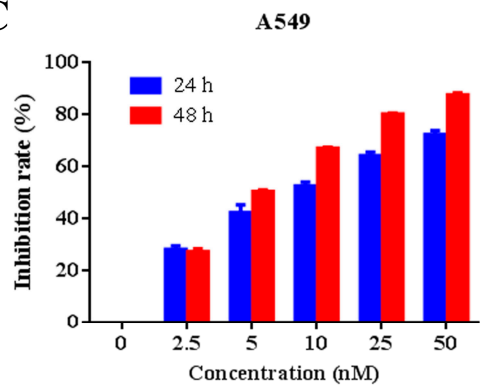

A549

$\mathbf{E}$

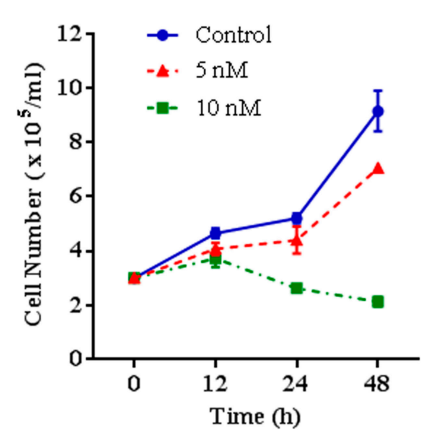

B

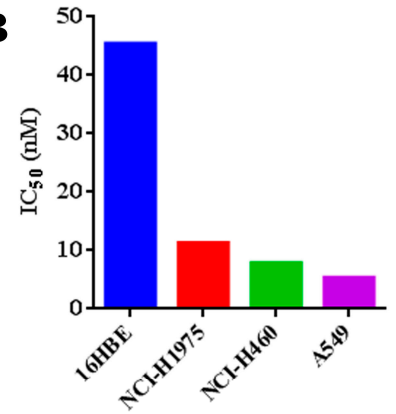

D

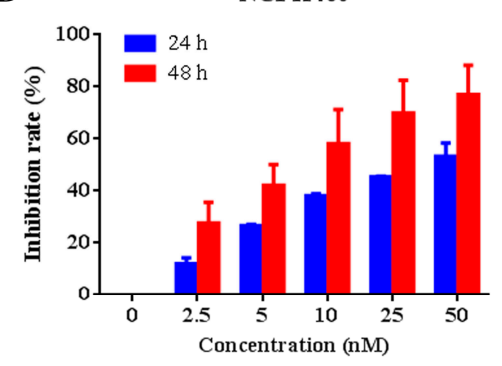

$\mathbf{F}$

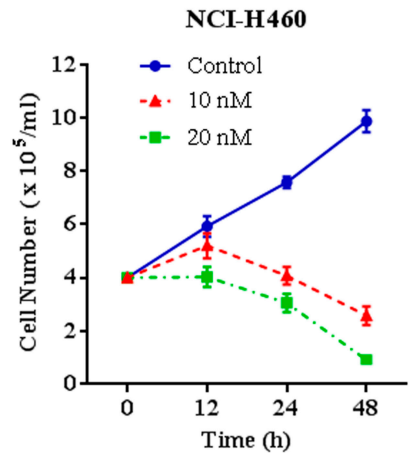

Figure 1. Arenobufagin decreases the cell viability in A549 and NCI-H460 cells. (A) The chemical structure of arenobufagin; (B) The $\mathrm{IC}_{50}$ values of arenobufagin for indicated cell lines; (C) The inhibitory effects of arenobufagin on A549 cells analyzed by 3-(4,5-dimethylthiazol-2-yl)-2,5-diphenyltetrazolium bromide (MTT) assay; (D) NCI-H460 cells were treated with various concentrations of arenobufagin for 24 and $48 \mathrm{~h}$. MTT assays were conducted to measure the cell viability; (E) A549 cells were treated with or without arenobufagin for indicated time points, and analyzed by trypan blue exclusion assay; (F) Inhibitory effects of arenobufagin on cell viability of NCI-H460 cells measured by trypan blue exclusion assay.

\subsection{Arenobufagin Provokes NSCLC Cell Apoptosis}

We next examined the potential effect of arenobufagin on NSCLC cell apoptosis. A549 and NCI-H460 cells were treated with the indicated doses of arenobufagin for $24 \mathrm{~h}$. Hoechst 33258 staining showed that arenobufagin-induced apoptotic chromatin condensation and DNA fragmentation were clearly observed (Figure 2A). We next detected the alteration of apoptosis-related proteins. As shown in Figure 2B,C, arenobufagin treatment induced a typical processing of caspase-9, caspase- 3 and a classical pattern of poly (ADP-ribose) polymerase (PARP) cleavage, while it had little effect on caspase- 8 cleavage in A549 and NCI-H460 cells. We also observed caspase-9 and PARP cleavage in NCI-H1975 cells (Supplementary, Figure S1C). Notably, A549 cell apoptosis, triggered by arenobufagin, was greatly inhibited after the pan-caspase inhibitor Z-VAD-FMK treatment, as revealed by Western blot assay and trypan blue exclusion assay (Figure 2D,E). These results indicated that arenobufagin induces caspase-dependent apoptotic death in NSCLC cells. 
A
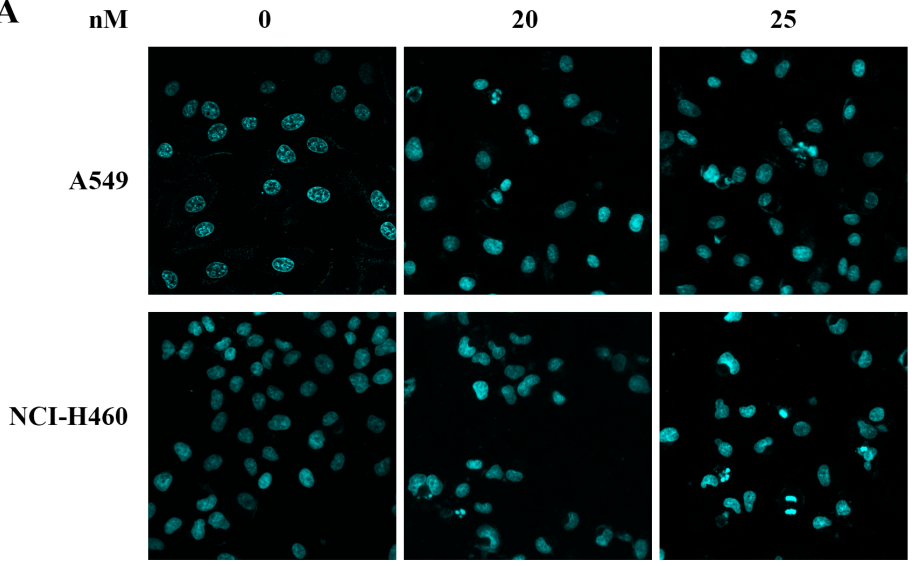

B

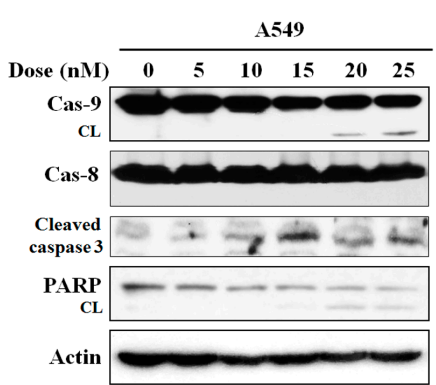

D

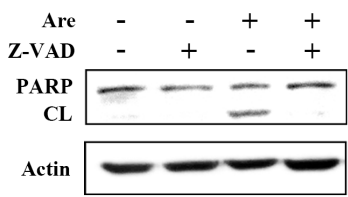

C

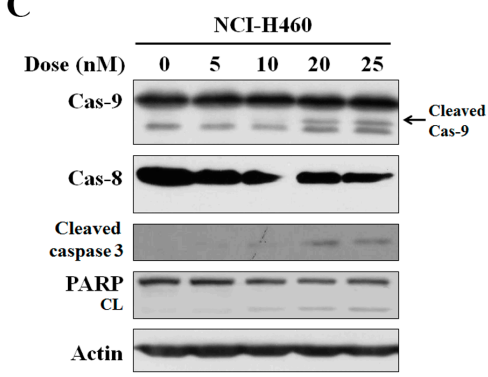

$\mathbf{E}$

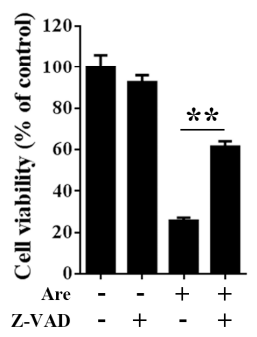

Figure 2. Arenobufagin induces caspase-dependent apoptosis in non-small-cell lung cancer (NSCLC) cells. (A) A549 and NCI-H460 cells were treated with the indicated concentrations of arenobufagin for $24 \mathrm{~h}$, and apoptotic morphological changes were evaluated by Hoechst 33258 staining; (B,C) A549 and NCI-H460 cells were treated with arenobufagin at the indicated concentrations for $24 \mathrm{~h}$, and protein extracts were subjected to Western blot assay with indicated antibodies; (D,E) A549 cells were pre-treated with the caspase inhibitor Z-VAD $(40 \mu \mathrm{M})$ for $1 \mathrm{~h}$, followed by incubation with arenobufagin (Are, $25 \mathrm{nM}$ ) for $24 \mathrm{~h}$; (D) poly (ADP-ribose) polymerase (PARP) cleavage was analyzed by Western blotting; (E) The cell viability was detected via trypan blue exclusion assay. ${ }^{* *} p<0.01$, arenobufagin-treated group versus arenobufagin and Z-VAD combination group.

\subsection{Arenobufagin Regulates Noxa and Mcl-1 in NSCLC Cells}

The data above showed that arenobufagin induced the cleavage of the caspase- 9 protein, which indicated that the intrinsic (mitochondria-mediated) apoptotic pathways were activated by arenobufagin. It was reported that the $\mathrm{Bcl}-2$ protein family represented the key regulatory node of mitochondrial apoptosis $[5,9]$. We then detected the expression of Bcl-2 family proteins. Interestingly, we found that after treatment with arenobufagin, Noxa protein, an important mediator of the mitochondrial apoptosis pathway, was significantly increased in A549 cells (Figure 3A). Early studies showed that Noxa had the most restricted potential to neutralize Mcl-1, and later evidence suggested that Noxa upregulation promoted the degradation of the Mcl-1 protein, an anti-apoptotic member of the Bcl-2 proteins family [11,13]. It was reported that modulation of Noxa and Mcl-1 was important for compound-induced anti-cancer effects [7,8,23]. We then detected a change of Mcl-1 in A549 
cells, and found that arenobufagin treatment dramatically downregulated the expression of Mcl-1 (Figure 3A). Arenobufagin also increased the expression of Noxa and abrogated Mcl-1 in NCI-H460 and NCI-H1975 cells, which were consistent with the results from A549 cells (Figure 3B,C). Further studies demonstrated that the upregulation of Noxa and reduction of Mcl-1 occurred within $6 \mathrm{~h}$, while caspase- 9 and PARP proteins were cleaved after $12 \mathrm{~h}$, indicating that the Noxa/Mcl-1 pathway was associated with arenobufagin-triggered apoptosis in NSCLC cells (Figure 3D).

A

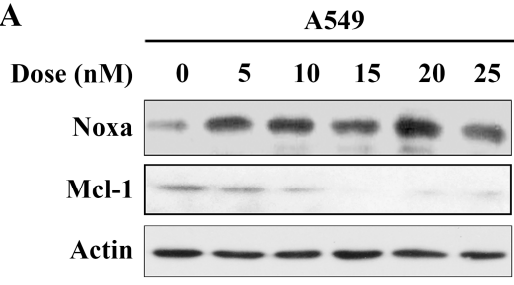

C

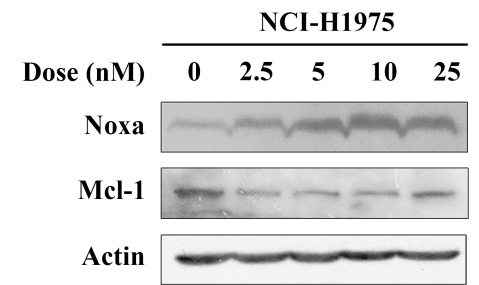

B

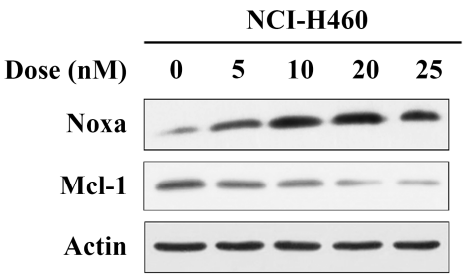

D

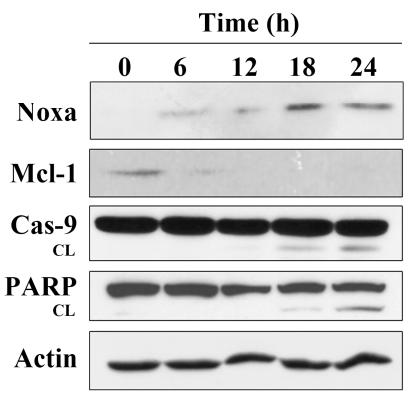

Figure 3. Arenobufagin up-regulates Noxa and down-regulates Mcl-1 in NSCLC cells. (A,B) A549 and NCI-H460 cells were incubated with the indicated concentrations of arenobufagin for $24 \mathrm{~h}$, and the protein expression levels of Noxa, Mcl-1, and Actin were determined by Western blotting. The level of actin was used as a loading control; (C) Noxa up-regulation and Mcl-1 down-regulation were determined using a Western blot analysis in NCI-H1975 cells; (D) A549 cells were treated with arenobufagin at $20 \mathrm{nM}$ for the indicated time points, and the expression of Noxa, Mcl-1, caspase-9, PARP and Actin were analyzed by Western blotting. The level of actin was used as a loading control.

\subsection{Noxa is Required for the Inhibition Effect of Arenobufagin on NSCLC Cells}

To further confirm the importance of Noxa in the arenobufagin-induced anti-NSCLC effect, we used Noxa siRNA to downregulate its expression. As shown in Figure 4A, siRNA dramatically downregulated the expression of Noxa in A549 cells. Using MTT assay, we confirmed that Noxa played a pivotal role in the function of arenobufagin (Figure 4B). In accordance with these results, PARP cleavage triggered by arenobufagin was also inhibited after a knockdown of Noxa (Figure 4C). The results above suggested that Noxa mediates the inhibitory effect of arenobufagin on NSCLC cells and plays an essential role in this process.

$\mathbf{A}$

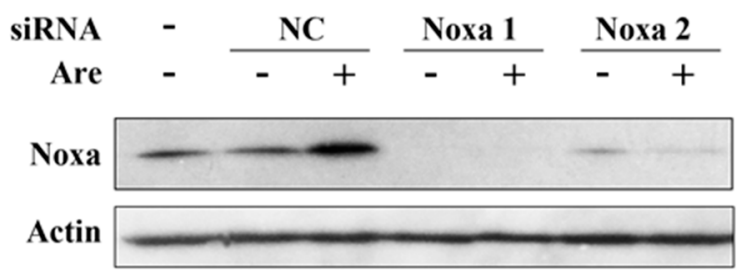

Figure 4. Cont. 
B

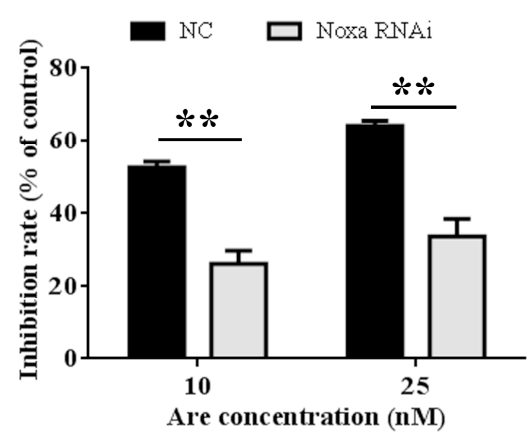

C

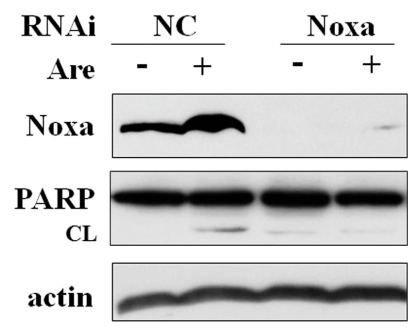

Figure 4. Noxa plays a critical role in the arenobufagin-induced inhibitory effect. (A) A549 cells transfected with control, or Noxa-specific siRNAs, for $48 \mathrm{~h}$ were treated with or without arenobufagin $(20 \mathrm{nM})$ for $24 \mathrm{~h}$, lysed, and Western blot analysis was performed; (B,C) A549 cells were transfected with siRNA and treated with arenobufagin as described in (A); (B) MTT assay was conducted to analyze the cell viability of A549 cells after Noxa siRNA transfection and the following arenobufagin treatment; (C) Western blot assay was used to measure the expression of Noxa and PARP. ** Indicates statistical significance $(p<0.01)$.

\section{5. $p 53$ is Involved in Arenobufagin-Induced Upregulation of Noxa}

Although we have shown that Noxa is upregulated after arenobufagin treatment in NSCLC cells, the mechanisms underlying this process are unclear. By using RT-PCR assay, we found that arenobufagin could upregulate Noxa at transcriptional levels in NSCLC cells (Figure 5A). Studies have shown that the transcription factors of p53, c-Myc and E2F1 can transcriptionally increase Noxa expression $[6,24,25]$. We then detected the expression of these proteins in NSCLC cells upon arenobufagin incubation. The results showed that p53 was significantly upregulated in NCI-H460 cells treated with arenobufagin (Figure 5B), while c-Myc and E2F1 were dramatically downregulated. This indicated that c-Myc and E2F1 were not the main mediators for the increase of Noxa that was triggered by arenobufagin. Thus, our results indicated that p53 may serve as a transcription factor for arenobufagin-induced Noxa upregulation. The possible mechanisms of arenobufagin-induced apoptosis in NSCLC cells can be summarized, as in Figure 5C.

A

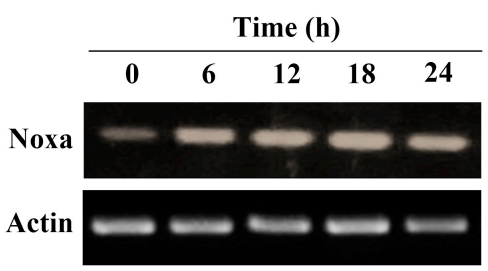

B

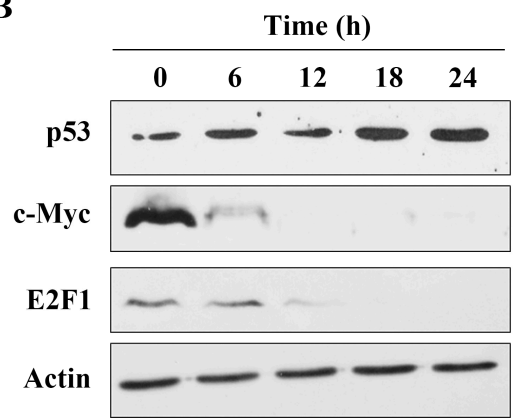

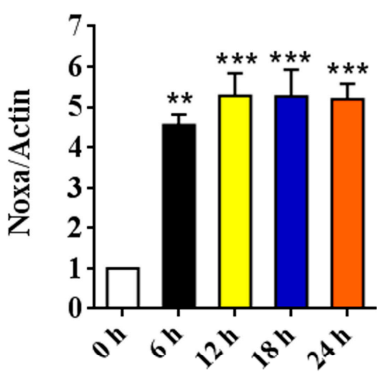

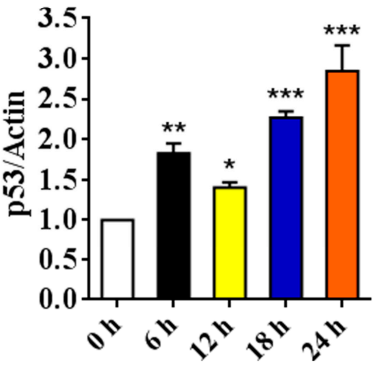

Figure 5. Cont. 


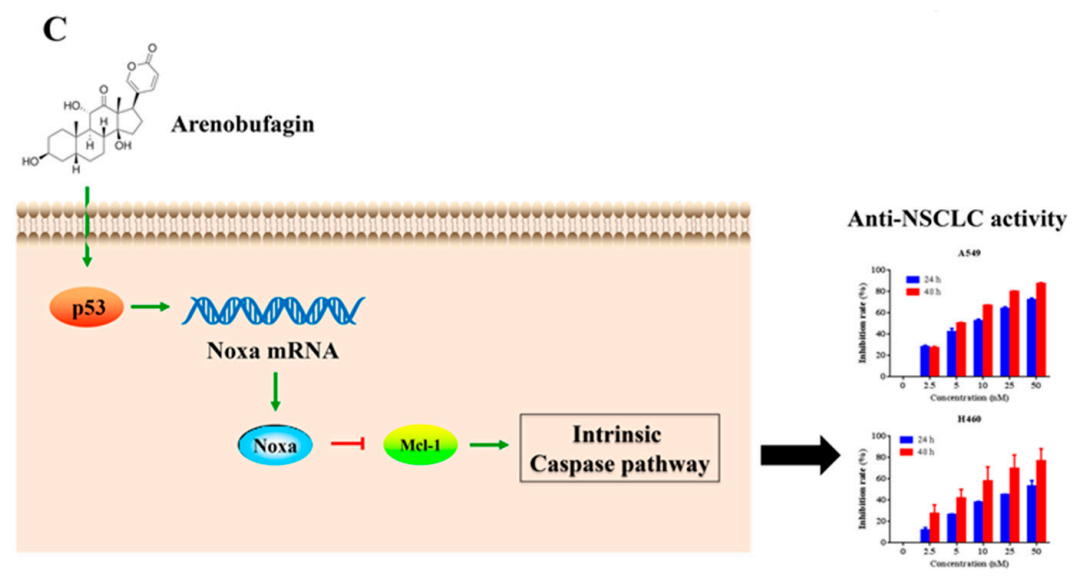

Figure 5. p53's role in arenobufagin-induced Noxa upregulation. (A) Reverse-Transcription PCR (RT-PCR) analysis of the expression of Noxa at the mRNA level in NCI-H460 cells treated with arenobufagin (25 nM) for indicated time points; (B) Western blot analysis of the protein expression of p53, c-Myc, and E2F1 in NCI-H460 cells treated with arenobufagin (25 nM) for indicated time points; (C) The schematic model of the molecular mechanisms of anti-NSCLC activity of arenobufagin. ${ }^{*} p<0.05$ compared with the control group; ${ }^{* *} p<0.01$ compared with the control group; ${ }^{* * *} p<0.001$ compared with the control group.

\section{Discussion}

Toad venom (also called $\mathrm{Chan} \mathrm{Su}$ ), initially recorded in traditional Chinese medicine more than 1000 years ago, is the dried secretion derived from either Bufo Bufo gargarizans Cantor, or Bufo melanostictus Suhneider. Toad venom has been widely used in China as a local anesthetic, cardiotonic, antimicrobial, and antineoplastic agent for many years [26,27]. Arenobufagin, as one of the main biologically active compounds extracted from toad venom, has been showed to exhibit potential anti-cancer effects in human hepatocellular carcinoma (HCC), esophageal squamous cell carcinoma and cervical carcinoma cells $[19,21,22]$. However, the anti-NSCLC effects of arenobufagin are largely unknown. In this study, we demonstrated for the first time that arenobufagin is able to inhibit growth and induce apoptosis in NSCLC cells, by modulation of the Noxa-related pathway, paving the way for this compound to be exploited as a therapeutic agent in the adjunct therapy of NSCLC.

Since the 1970s, Chinese clinical trials have repeatedly shown that Huachansu, an injectable form of toad venom in physiological saline solution, has anticancer activity with low toxicity and mild adverse effects. Using a phase I clinical trial design, Meng et al. reported that no dose-limiting toxicities (DLT) were observed with the use of Huachansu at doses up to eight times higher than typically used in China [26]. As for the selective activity of a single compound from the toad venom, Lv et al. reported that arenobufagin showed lower toxicity towards human normal esophageal squamous cells, compared with esophageal squamous cell carcinoma (ESCC) cells [22]. Takai et al. also showed that bufalin, another active component of toad venom, had inhibition effects on human endometrial and ovarian cancer cells, with little toxic effect on normal cells at low doses [28]. In accordance with these studies, we found that arenobufagin significantly inhibited the growth of NSCLC cells, while displaying lower toxicity towards 16HBE normal human bronchial epithelial cells. This indicated a preference of arenobufagin in inhibiting NSCLC cells over normal human bronchial epithelial cells.

Researchers showed that arenobufagin induced apoptosis in hepatocellular carcinoma, esophageal squamous cell carcinoma and cervical carcinoma cells [19,21,22]. Our data demonstrated that arenobufagin also induced apoptosis in NSCLC cells. It was reported that the mechanisms of arenobufagin-induced apoptosis were implicated in the PI3K/Akt/mTOR pathways and the p53 pathway $[19,22]$. In this study, we found a novel mechanism that arenobufagin could induce mitochondria-mediated apoptosis in NSCLC cells via regulation of Noxa-related pathways. Noxa 
and Mcl-1 are members of Bcl-2 protein family. Noxa is well known for its pro-apoptotic effect, while Mcl-1 is a classic anti-apoptotic protein. They have opposing apoptotic activities that mediate cell death. The importance of Noxa and Mcl-1 as drug targets in cancer therapy is becoming increasingly evident. Our results indicated that upregulation of Noxa and decrease of Mcl-1 were responsible for the pro-apoptosis effect of arenobufagin. In accordance with our findings, research has shown that the modulation of Noxa and Mcl-1 is critical for the cytotoxic effect of many anticancer treatments. Therefore, our study implied that targeting the Noxa/Mcl-1 pathway could serve as a new treatment strategy for NSCLC therapy.

Noxa was initially identified as a primary p53-responsive gene, and could be regulated transcriptionally in response to genotoxic stress [6]. Recently, Lv et al. reported that arenobufagin moderately increased the expression of the p53 protein and significantly enhanced its phosphorylation in ESCC cells [22]. Interestingly, we found that arenobufagin also increased p53, and that the activation of p53 might be involved in arenobufagin-induced upregulation of Noxa in NSCLC cells. Noxa appears to be crucial for fine-tuning cell death decisions by targeting the Mcl-1 protein for degradation. This event appears to be critical for cell death induction along the mitochondrial Bcl2-regulated apoptosis pathway, in response to factor deprivation or DNA damage $[10,29,30]$. P53 was widely reported to be potentially activated by DNA damage [31,32]. A recent study also showed that arenobufagin intercalated with DNA and induced DNA damage, as well as a transient increase in transcriptionally active p53 in HCC cells [20]. Therefore, our results suggested that arenobufagin might regulate the p53/Noxa/Mcl-1 pathway (Figure 5C).

\section{Materials and Methods}

\subsection{Cell Lines and Cell Culture}

The lung cancer lines NCI-H1975, A549 and NCI-H460 were obtained from the American Tissue Culture Collection (ATCC). Human normal bronchial epithelial cell line 16HBE was purchased from the Cell Resource Center, Chinese Academy of Medical Sciences (Beijing, China) and cultured according to standard protocols. The cells were grown in a humidified incubator containing $5 \% \mathrm{CO}_{2}$ in air at $37^{\circ} \mathrm{C}$. A549 and NCI-H460 cells were cultured with Dulbecco's Modified Eagle's Medium (DMEM, HyClone, Logan, UT, USA) while NCI-H1975 cells were cultured in Roswell Park Memorial Institute (RPMI)-1640 (HyClone, Logan, UT, USA). Both media were supplemented with 10\% fetal bovine serum (FBS, HyClone, Logan, UT, USA), $100 \mathrm{U} / \mathrm{mL}$ penicillin and $100 \mathrm{mg} / \mathrm{mL}$ streptomycin.

\subsection{Reagents and Antibodies}

Arenobufagin was purchased from MedChem Express (MedChem Express, Shanghai, China) and dissolved in dimethylsulfoxide (DMSO, Vetec) to make a stock solution at $50 \mathrm{mM}$ and stored at $-20^{\circ} \mathrm{C}$ until used. The caspase inhibitor Z-VAD-fmk and antibodies including anti-cleaved caspase- 3 and caspase- 8 were obtained from the Beyotime Institute of Biotechnology (Haimen, China). The small interfering RNAs (siRNAs) were synthesized by Shanghai GenePharma Co. Ltd (Shanghai, China). Lipofectamine 2000 reagent was purchased from Invitrogen. The anti-PARP and caspase-9 antibodies were obtained from Cell Signaling Technology (Danvers, MA, USA). The antibodies including anti-Noxa, Mcl-1, p53 and c-Myc, and the secondary antibodies, including horseradish peroxidases (HRP)-conjugated goat anti-mouse and goat anti-rabbit immunoglobulin G were purchased from Santa Cruz (Dallas, TX, USA). Human E2F1 and actin antibodies were purchased from Sigma-Aldrich.

\subsection{Cell Viability Assay}

Cell viability was measured by the MTT cytotoxicity assay [33]. A549 and NCI-H460 cells were seeded into 96-well plates at a density of 5000-10,000 cells per well, with three replicate wells per group. When the cells of each well reached $70-80 \%$ confluent, the indicated concentrations of arenobufagin were added. After treatment for 24 and $48 \mathrm{~h}$, the cells were incubated with $10 \mu \mathrm{L}$ of MTT $(5 \mathrm{mg} / \mathrm{mL})$ 
for $2-4 \mathrm{~h}$ at $37^{\circ} \mathrm{C}$. Then, the medium was discarded and $150 \mu \mathrm{L}$ of DMSO was added to each well to dissolve formazan for measurement. The optical density (OD) was measured at an absorbance wavelength of $490 \mathrm{~nm}$, using a microplate reader (Thermo Fisher, Waltham, MA, USA).

\subsection{Hoechst 33258 Staining}

Characteristic apoptotic morphological changes were assessed by Hoechst 33258 staining, using the Hoechst staining kit (Beyotime, Haimen, China). In brief, cells were exposed to arenobufagin for $24 \mathrm{~h}$ and then fixed with fixing buffer for $10 \mathrm{~min}$ at room temperature. After staining with Hoechst 33258 for $10 \mathrm{~min}$, the cells were observed under a confocal laser scanning microscope (Fluoview FV10i, Olympus, Tokyo, Japan) at high magnification $(600 \times)$ to determine nuclei fragmentation and chromatin condensation.

\subsection{Western Blot Analysis}

After treatments, cells were lysed on ice for $30 \mathrm{~min}$ in RIPA buffer containing $50 \mathrm{mM}$ Tris- $\mathrm{HCl}$

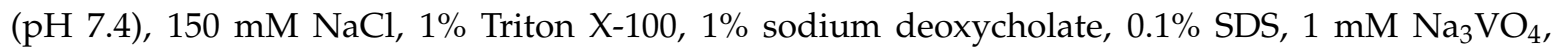
$1 \mathrm{mM} \mathrm{NaF}$, and $1 \mathrm{mM}$ PMSF. Then, lysates were centrifuged at $12,000 \times \mathrm{g}$ for $10 \mathrm{~min}$ at $4{ }^{\circ} \mathrm{C}$ to obtain the supernatant extract, followed by quantitation with the bicinchoninic acid (BCA) method. After denaturation, an equal quantity of total protein per lane was separated by sodium dodecyl sulfate-polyacrylamide gel electrophoresis (SDS-PAGE), and transferred to nitrocellulose membranes. The membranes were blocked with 5\% no-fat milk in Tris-buffered saline containing Tween 20 (TBST) for $1 \mathrm{~h}$ at room temperature and incubated with specific primary antibodies overnight at $4{ }^{\circ} \mathrm{C}$. Following four washes ( 5 min each) with TBST, the membranes were incubated with the corresponding secondary antibody for another $2 \mathrm{~h}$ at room temperature. After extensive washing with TBST, the signals on the membranes were detected using an enhanced chemiluminescence substrate (Cwbiotech, Beijing, China). Actin served as an endogenous loading control.

\subsection{RNA Interference}

Cells $(2 \times 105$ cells /well) were seeded in 6-well plates, and transfected with Noxa or negative control (NC) siRNA using lipofectamine 2000 following the manufacturer's instructions. After $48 \mathrm{~h}$ transfection, the cells were treated with arenobufagin for an additional $24 \mathrm{~h}$, followed by the Western blot and MTT assay, as previously described [34]. The siRNA sequences of Noxa were as follows: 5'-GGUGCACGUUUCAUCAAUU-3' (Noxa 1), 5'-GGAGAUUUGGAGACAAACU-3' (Noxa 2) [25,35].

\subsection{RNA Extraction and Reverse-Transcription PCR}

According to the manufacturer's protocol, the total RNA of the cells was extracted using the TRIZOL Reagent (Invitrogen, Carlsbad, CA, USA) and the phenol-chloroform extraction method. cDNA synthesis was performed using PrimeScriptTM 1st Strand cDNA Synthesis Kit (TaKaRa). Primers for Noxa detection were as follows: sense primer: 5'-AAGAAGGCGCGCAAGAAC-3'; antisense primer: 5'-CGTGCACCTCCTGAGAAAAC-3' [25]. The reaction mix contained: $2.5 \mu \mathrm{L}$ $10 \times$ Ex Taq Buffer, $2 \mu \mathrm{L}$ dNTP Mixture, $200 \mathrm{nM}$ forward and reverse primers, $100 \mathrm{ng}$ cDNA template, $0.25 \mu \mathrm{L}$ TaKaRa Ex Taq and $\mathrm{ddH}_{2} \mathrm{O}$ up to $25 \mu \mathrm{L}$ volume. The PCR cycling conditions consisted of the following: $98{ }^{\circ} \mathrm{C}$ for $10 \mathrm{~s}$ for denaturation, $55^{\circ} \mathrm{C}$ for $15 \mathrm{~s}$ for annealing and $72{ }^{\circ} \mathrm{C}$ for $30 \mathrm{~s}$ for extension, for a total of 30 cycles. Products of RT-PCR were separated by $1.5 \%$ agarose gel electrophoresis and detected in a gel imaging system (UVP GelMax Imager System, Upland, CA, USA).

\subsection{Statistical Analysis}

Data were collected and analyzed using GraphPad Prism 6.0 software and expressed as the mean \pm standard deviation (SD). Student's $t$-test was used to compare data between two groups. 
One way analysis of variance was performed to compare data of more than two groups. A value of $p<$ 0.05 was considered to be statistically significant.

\section{Conclusions}

In summary, we demonstrated that arenobufagin inhibited growth and induced apoptosis in NSCLC cells. Mechanistically, we found that the activation of Noxa-related pathways might contribute to the anti-NSCLC effects of arenobufagin. Therefore, our study demonstrates that arenobufagin exhibits potent activity against NSCLC cells through a novel mechanism, which will be beneficial for the application of this compound to the treatment of NSCLC.

Supplementary Materials: Supplementary materials are available online.

Acknowledgments: We thank Xiaoyan Sun (Laboratory of Cellular and Molecular Biology, Jiangsu Province Academy of Traditional Chinese Medicine, Nanjing, China) for help with the Hoechst 33258 staining experiment. This study was supported by the National Natural Science Foundation of China (Nos. 81402511 and 81201577) and the Student Research Training Program of Anhui University of Technology (Nos. 201510360171 and 2015024Z).

Author Contributions: L.M., X.L. and Z.L. conceived and designed the experiments; L.M., Y.Z., S.F., H.L. performed the experiments; L.M., X.L. and Z.L. analyzed the data; X.L. contributed reagents/materials/analysis tools; L.M. and Z.L. wrote the paper.

Conflicts of Interest: The authors declare no conflict of interest.

\section{References}

1. Ferlay, J.; Soerjomataram, I.; Dikshit, R.; Eser, S.; Mathers, C.; Rebelo, M.; Parkin, D.M.; Forman, D.; Bray, F. Cancer incidence and mortality worldwide: Sources, methods and major patterns in GLOBOCAN 2012. Int. J. Cancer 2015, 136, E359-E386. [CrossRef] [PubMed]

2. Molina, J.R.; Yang, P.; Cassivi, S.D.; Schild, S.E.; Adjei, A.A. Non-small cell lung cancer: Epidemiology, risk factors, treatment, and survivorship. Mayo Clin. Proc. 2008, 83, 584-594. [CrossRef]

3. Hirsch, F.R.; Scagliotti, G.V.; Mulshine, J.L.; Kwon, R.; Curran, W.J., Jr.; Wu, Y.L.; Paz-Ares, L. Lung cancer: Current therapies and new targeted treatments. Lancet 2017, 389, 299-311. [CrossRef]

4. Bai, L.; Wang, S. Targeting apoptosis pathways for new cancer therapeutics. Annu. Rev. Med. 2014, 65, 139-155. [CrossRef] [PubMed]

5. Cory, S.; Huang, D.C.; Adams, J.M. The Bcl-2 family: Roles in cell survival and oncogenesis. Oncogene 2003, 22, 8590-8607. [CrossRef] [PubMed]

6. Oda, E.; Ohki, R.; Murasawa, H.; Nemoto, J.; Shibue, T.; Yamashita, T.; Tokino, T.; Taniguchi, T.; Tanaka, N. Noxa, a BH3-only member of the Bcl-2 family and candidate mediator of p53-induced apoptosis. Science 2000, 288, 1053-1058. [CrossRef] [PubMed]

7. Simonin, K.; N'Diaye, M.; Lheureux, S.; Loussouarn, C.; Dutoit, S.; Briand, M.; Giffard, F.; Brotin, E.; Blanc-Fournier, C.; Poulain, L. Platinum compounds sensitize ovarian carcinoma cells to ABT-737 by modulation of the Mcl-1/Noxa axis. Apoptosis 2013, 18, 492-508. [CrossRef] [PubMed]

8. Lucas, K.M.; Mohana-Kumaran, N.; Lau, D.; Zhang, X.D.; Hersey, P.; Huang, D.C.; Weninger, W.; Haass, N.K.; Allen, J.D. Modulation of NOXA and MCL-1 as a strategy for sensitizing melanoma cells to the BH3-mimetic ABT-737. Clin. Cancer Res. 2012, 18, 783-795. [CrossRef] [PubMed]

9. Albert, M.C.; Brinkmann, K.; Kashkar, H. Noxa and cancer therapy: Tuning up the mitochondrial death machinery in response to chemotherapy. Mol. Cell. Oncol. 2014, 1, e29906. [CrossRef] [PubMed]

10. Yan, J.; Zhong, N.; Liu, G.; Chen, K.; Liu, X.; Su, L.; Singhal, S. Usp9x- and Noxa-mediated Mcl-1 downregulation contributes to pemetrexed-induced apoptosis in human non-small-cell lung cancer cells. Cell Death Dis. 2014, 5, e1316. [CrossRef] [PubMed]

11. Chen, L.; Willis, S.N.; Wei, A.; Smith, B.J.; Fletcher, J.I.; Hinds, M.G.; Colman, P.M.; Day, C.L.; Adams, J.M.; Huang, D.C. Differential targeting of prosurvival Bcl-2 proteins by their BH3-only ligands allows complementary apoptotic function. Mol. Cell 2005, 17, 393-403. [CrossRef] [PubMed]

12. Gomez-Bougie, P.; Menoret, E.; Juin, P.; Dousset, C.; Pellat-Deceunynck, C.; Amiot, M. Noxa controls Mule-dependent Mcl-1 ubiquitination through the regulation of the Mcl-1/USP9X interaction. Biochem. Biophys. Res. Commun. 2011, 413, 460-464. [CrossRef] [PubMed] 
13. Willis, S.N.; Chen, L.; Dewson, G.; Wei, A.; Naik, E.; Fletcher, J.I.; Adams, J.M.; Huang, D.C. Proapoptotic Bak is sequestered by Mcl-1 and Bcl-xL, but not Bcl-2, until displaced by BH3-only proteins. Genes Dev. 2005, 19, 1294-1305. [CrossRef] [PubMed]

14. Liu, Z.; Ma, L.; Zhou, G.B. The main anticancer bullets of the Chinese medicinal herb, thunder god vine. Molecules 2011, 16, 5283-5297. [CrossRef] [PubMed]

15. Garraffo, H.M.; Gros, E.G. Biosynthesis of bufadienolides in toads. VI. Experiments with [1,H]cholesterol, [24C]coprostanol, and 5 beta-[24C]pregnanolone in the toad Bufo arenarum. Steroids 1986, 48, 251-257. [CrossRef]

16. Li, M.; Wu, S.; Liu, Z.; Zhang, W.; Xu, J.; Wang, Y.; Liu, J.; Zhang, D.; Tian, H.; Li, Y.; Ye, W. Arenobufagin, a bufadienolide compound from toad venom, inhibits VEGF-mediated angiogenesis through suppression of VEGFR-2 signaling pathway. Biochem. Pharmacol. 2012, 83, 1251-1260. [CrossRef] [PubMed]

17. Cruz Jdos, S.; Matsuda, H. Arenobufagin, a compound in toad venom, blocks $\mathrm{Na}\left({ }^{+}\right)-\mathrm{K}^{+}$pump current in cardiac myocytes. Eur. J. Pharmacol. 1993, 239, 223-226. [CrossRef]

18. Cruz Jdos, S.; Matsuda, H. Depressive effects of arenobufagin on the delayed rectifier $\mathrm{K}^{+}$current of guinea-pig cardiac myocytes. Eur. J. Pharmacol. 1994, 266, 317-325. [CrossRef]

19. Zhang, D.M.; Liu, J.S.; Deng, L.J.; Chen, M.F.; Yiu, A.; Cao, H.H.; Tian, H.Y.; Fung, K.P.; Kurihara, H.; Pan, J.X.; et al. Arenobufagin, a natural bufadienolide from toad venom, induces apoptosis and autophagy in human hepatocellular carcinoma cells through inhibition of PI3K/Akt/mTOR pathway. Carcinogenesis 2013, 34, 1331-1342. [CrossRef] [PubMed]

20. Deng, L.J.; Peng, Q.L.; Wang, L.H.; Xu, J.; Liu, J.S.; Li, Y.J.; Zhuo, Z.J.; Bai, L.L.; Hu, L.P.; Chen, W.M.; et al. Arenobufagin intercalates with DNA leading to G2 cell cycle arrest via ATM/ATR pathway. Oncotarget 2015, 6, 34258-34275. [PubMed]

21. Yue, Q.; Zhen, H.; Huang, M.; Zheng, X.; Feng, L.; Jiang, B.; Yang, M.; Wu, W.; Liu, X.; Guo, D. Proteasome Inhibition Contributed to the Cytotoxicity of Arenobufagin after Its Binding with Na, K-ATPase in Human Cervical Carcinoma HeLa Cells. Plos One 2016, 11, e0159034. [CrossRef] [PubMed]

22. Lv, J.; Lin, S.; Peng, P.; Cai, C.; Deng, J.; Wang, M.; Li, X.; Lin, R.; Lin, Y.; Fang, A.; et al. Arenobufagin activates p53 to trigger esophageal squamous cell carcinoma cell apoptosis in vitro and in vivo. Onco Targets Ther. 2017, 10, 1261-1267. [CrossRef] [PubMed]

23. Tromp, J.M.; Geest, C.R.; Breij, E.C.; Elias, J.A.; van Laar, J.; Luijks, D.M.; Kater, A.P.; Beaumont, T.; van Oers, M.H.; Eldering, E. Tipping the Noxa/Mcl-1 balance overcomes ABT-737 resistance in chronic lymphocytic leukemia. Clin. Cancer Res. 2012, 18, 487-498. [CrossRef] [PubMed]

24. Hershko, T.; Ginsberg, D. Up-regulation of Bcl-2 homology 3 (BH3)-only proteins by E2F1 mediates apoptosis. J. Biol. Chem. 2004, 279, 8627-8634. [CrossRef] [PubMed]

25. Zhang, B.; Jiao, J.; Liu, Y.; Guo, L.X.; Zhou, B.; Li, G.Q.; Yao, Z.J.; Zhou, G.B. Gefitinib analogue V1801 induces apoptosis of T790M EGFR-harboring lung cancer cells by up-regulation of the BH-3 only protein Noxa. PLoS ONE 2012, 7, e48748. [CrossRef] [PubMed]

26. Meng, Z.; Yang, P.; Shen, Y.; Bei, W.; Zhang, Y.; Ge, Y.; Newman, R.A.; Cohen, L.; Liu, L.; Thornton, B.; et al. Pilot study of huachansu in patients with hepatocellular carcinoma, nonsmall-cell lung cancer, or pancreatic cancer. Cancer 2009, 115, 5309-5318. [CrossRef] [PubMed]

27. Qi, F.; Li, A.; Inagaki, Y.; Kokudo, N.; Tamura, S.; Nakata, M.; Tang, W. Antitumor activity of extracts and compounds from the skin of the toad Bufo bufo gargarizans Cantor. Int. Immunopharmacol. 2011, 11, 342-349. [CrossRef] [PubMed]

28. Takai, N.; Ueda, T.; Nishida, M.; Nasu, K.; Narahara, H. Bufalin induces growth inhibition, cell cycle arrest and apoptosis in human endometrial and ovarian cancer cells. Int. J. Mol. Med. 2008, 21, 637-643. [CrossRef] [PubMed]

29. Ploner, C.; Kofler, R.; Villunger, A. Noxa: At the tip of the balance between life and death. Oncogene 2008, 27 (Suppl. 1), S84-S92. [CrossRef] [PubMed]

30. Nakajima, W.; Sharma, K.; Lee, J.Y.; Maxim, N.T.; Hicks, M.A.; Vu, T.T.; Luu, A.; Yeudall, W.A.; Tanaka, N.; Harada, H. DNA damaging agent-induced apoptosis is regulated by MCL-1 phosphorylation and degradation mediated by the Noxa/MCL-1/CDK2 complex. Oncotarget 2016, 7, 36353-36365. [CrossRef] [PubMed]

31. Roos, W.P.; Thomas, A.D.; Kaina, B. DNA damage and the balance between survival and death in cancer biology. Nat. Rev. Cancer 2016, 16, 20-33. [CrossRef] [PubMed] 
32. Pearl, L.H.; Schierz, A.C.; Ward, S.E.; Al-Lazikani, B.; Pearl, F.M. Therapeutic opportunities within the DNA damage response. Nat. Rev. Cancer 2015, 15, 166-180. [CrossRef] [PubMed]

33. Ma, L.; Wen, Z.S.; Liu, Z.; Hu, Z.; Ma, J.; Chen, X.Q.; Liu, Y.Q.; Pu, J.X.; Xiao, W.L.; Sun, H.D.; et al. Overexpression and small molecule-triggered downregulation of CIP2A in lung cancer. PLoS ONE 2011, 6, e20159. [CrossRef] [PubMed]

34. Liu, Z.; Ma, L.; Wen, Z.S.; Hu, Z.; Wu, F.Q.; Li, W.; Liu, J.; Zhou, G.B. Cancerous inhibitor of PP2A is targeted by natural compound celastrol for degradation in non-small-cell lung cancer. Carcinogenesis 2014, 35, 905-914. [CrossRef] [PubMed]

35. Smith, A.J.; Dai, H.; Correia, C.; Takahashi, R.; Lee, S.H.; Schmitz, I.; Kaufmann, S.H. Noxa/Bcl-2 protein interactions contribute to bortezomib resistance in human lymphoid cells. J. Biol. Chem. 2011, 286, 17682-17692. [CrossRef] [PubMed]

Sample Availability: Samples of the compounds are available from the authors.

(C) 2017 by the authors. Licensee MDPI, Basel, Switzerland. This article is an open access article distributed under the terms and conditions of the Creative Commons Attribution (CC BY) license (http:/ / creativecommons.org/licenses/by/4.0/). 